Pacific Journal of Mathematics

A DECISION PROCEDURE FOR A CLASS OF FORMULAS OF 


\title{
A DECISION PROCEDURE FOR A CLASS OF FORMULAS OF FIRST ORDER PREDICATE CALCULUS
}

\author{
M. R. KROM
}

1. Introduction. In [4] (c.f. also case $V^{\prime}$ page 256 of [1]) J. Herbrand provides a decision procedure which is equivalent to a decision procedure for determining for a fixed contradiction $C$ and for any first order prenex formula $\Gamma$ whose matrix is a conjunction of signed atomic formulas, whether $\Gamma \rightarrow C$ is valid. In this paper we define a class a of first order formulas and then provide a decision procedure for determining for any first order prenex formula $\Gamma$ whose matrix is a conjunction of signed atomic formulas and and member $\Delta$ of the class, whether $\Gamma \rightarrow \Delta$ is valid. Although the class of formulas $\Delta$ that we consider is rather large, it is clear that some restriction is necessary since a decision procedure for the class itself is obtained by using for $\Gamma$ a single propositional parameter that does not occur in 4 .

The formulas we consider are those of any system of pure first order predicate calculus without equality and without function symbols. We use $\vee, \wedge, \neg$, and $\rightarrow$ for the propositional connectives disjunction, conjunction, denial, and the conditional, respectively. The symbols $\Gamma, \Delta, \Gamma_{0}, \Delta_{0}, \Gamma_{1}, \Delta_{1}, \cdots$ shall range over arbitrary formulas, $P, Q, P_{1}, Q_{1}, \cdots$ over prefixes, and $M, N, M_{1}, N_{1}, \cdots$ over matrices. A propositional parameter or predicate parameter together with its attached individual variables or individual parameters will be called an atomic formula. An occurrence of an atomic formula in a formula $\Gamma$ is called an atomic part of $\Gamma$. Two prenex formulas are similar if their matrices differ only in the symbols occupying individual variable places of the atomic formulas. Two prenex formulas are congruent if they differ only by equivalent replacements of bound variables. We indicate that $\Delta$ is a logical consequence of $\Gamma$ by writing $\Gamma \models \Delta$. If $\Gamma \models \Delta$ then there exists a symmetric $L$-deduction of $\Delta$ from $\Gamma$ as described in [2]. For any formulas $\Gamma$ and $\Delta$ an $L$-deduction of $\Delta$ from $\Gamma$ is an ordered $(n+1)$ tuple $\left\langle\Gamma_{0}, \cdots, \Gamma_{n}\right\rangle$ where $\Gamma_{0}=\Gamma$ and $\Gamma_{n}=\Delta$, together with a specification of how, for any $m<n, \Gamma_{m+1}$ results from $\Gamma_{m}$ by an application of an $L$-rule. The reader is referred to pages 252 and 253 of [2] for the definitions of the eleven $L$-rules. An $L$-deduction is symmetric if and only if the order in which the different kinds of $L$-rules are applied satisfies conditions (iii) through (vi) on page 257 of [2]. In addition, for convenience, we require that a symmetric $L$-deduction have exactly one application of the operation matrix change.

Received June 6, 1963. The author is grateful to the referee for many valuable suggestions. 
Our method for deciding whether $\Gamma \rightarrow \Delta$ is valid will be to determine whether there exists an $L$-deduction of $\Delta$ from $\Gamma$. We begin by describing the class of the formulas $\Delta$ for which our method applies.

2. Negation distinguished formulas. A Negation Distinguished formula (we will write $N D$ formula) is a prenex formula $\Delta$ whose matrix is in disjunctive normal form such that for any two occurrences $\bar{A}, \bar{B}$ in $A$, one positive and the other negative, of atomic formulas $A, B$ containing the same predicate parameter: (1) If $A=B$, then quantifier occurrences in 4 , if any, binding a variable in $\bar{A}, \bar{B}$ the rightmost is universal; and (2) If $A \neq B$, then some place in $\bar{A}$ and the corresponding place in $\bar{B}$ contain occurrences of different individual symbols such that neither occurrence is existentially bound in $\Delta$. In order to establish some properties of $N D$ formulas we make some observations about Linear Reasoning. Let $\mathscr{D}$ be a symmetric $L$-deduction of a prenex disjunctive formula $\Delta$ from a prenex formula $\Gamma$. For any formula ${ }^{1} A_{1}$ of $\mathscr{D}$ to which the operation simplication is applied the occurrence of the connective $V$ between the subformulas of $A_{1}$ that are combined in the simplication will be called the center of the formula $\Delta_{1}$. For any atomic part of any formula occurring after the matrix change in $\mathscr{D}$ we will define exactly one successor atomic part in any subsequent formula of $\mathscr{D}$. Let $\Delta_{1}$ and $\Delta_{2}$ be two formulas occurring after the matrix change in $\mathscr{D}$ such that $\Delta_{2}$ occurs immediately after $\Delta_{1}$. The successor in $\Delta_{2}$ of an atomic part of $\Delta_{1}$ is the atomic part of $A_{2}$ in the same relative position, where in the case of an application of simplication the position is determined by counting from the left for an atomic part left of the center and by counting from the right for an atomic part right of the center. The successor in any later formula of an atomic part is defined as required so that the relation of successor is the smallest transitive relation including the members described above. Similarly, for any atomic part of a formula occurring before the matrix change in $\mathscr{D}$ there is exactly one predecessor atomic part of any earlier formula of $\mathscr{D}$.

Let $\Delta_{1}$ and $\Delta_{2}$ be two formulas occurring after the matrix change in $\mathscr{D}$ such that $\Delta_{2}$ occurs immediately after $\Delta_{1}$. If $\Delta_{2}$ is obtained from $\Delta_{1}$ by an application of simplication then the relation of successor between the atomic parts of $\Delta_{1}$ and the atomic parts of $\Delta_{2}$ is two-to-one for atomic parts of the subformulas of $\Delta_{1}$ that are combined by the simplication. For any other atomic parts of $A_{1}$ the relation of successor is one-to-one. Also if $A_{2}$ is obtained from $A_{1}$ by an $L$-operation other

${ }^{1}$ We use the expression "formula of $\mathscr{D}$ " rather than the more cumbersome "occurrence of a formula of $\mathscr{D} "$ for convenience. Actually, we could assume that all formulas of an $L$-deduction are distinct. 
than simplication then the relation of successor between the atomic parts of $A_{1}$ and the atomic parts of $A_{2}$ is one-to-one.

Let $\Delta_{1}$ be a formula occurring after the matrix change in $\mathscr{D}$, let $\Delta_{2}$ be any later formula of $\mathscr{D}$, and let $\bar{A}_{1}$ and $\bar{B}_{1}$, be atomic parts of $\Delta_{1}$ such that no individual variables in their successors $\bar{A}_{2}$ and $\bar{B}_{2}$, respectively, in $\Delta_{2}$ are bound by a quantifier occurrence that is not in the main prefix of $A_{2}$ (c.f. page 254 of [2]). It follows from the properties of the $L$-rules that could be applied in the subdeduction of $\mathscr{D}$ of $\Delta_{2}$ from $\Delta_{1}$ that if $\bar{A}_{2}$ and $\bar{B}_{2}$ are occurrences of the same atomic formula then so are $\bar{A}_{1}$ and $\bar{B}_{1}$. Because of this property of symmetric $L$-deductions we may assume that for any application of the operation simplication the subformulas combined in the simplication include at least one quantifier. That is, simplications combining quantifier free subformulas (we will call these trivial simplications) can be avoided by deleting one or more of some identical disjuncts in the matrix of the formula resulting from matrix change in $\mathscr{D}$. Let $\bar{\Delta}_{1}^{1}$ be a subformula of $\Delta_{1}$ consisting of an occurrence of a prenex formula $\Delta_{1}^{1}$ in $\Delta_{1}$ such that there is a quantifier occurrence in $\bar{\Delta}_{1}^{1}$ which is not a quantifier occurrence in the main prefix of $\Delta_{1}$ and such that any quantifier occurrence of $\Delta_{1}$ which is not in $\bar{\Delta}_{1}^{1}$ and with $\bar{\Delta}_{1}^{1}$ in its scope is in the main prefix of $A_{1}$. We will say that $\bar{\Delta}_{1}^{1}$ is a proper prenex subformula of $A_{1}$. It follows from properties of the disassembling operations that $\Delta_{1}^{1}$ is similar to $A$.

We assumed that the concluding formula $\Delta$ of $\mathscr{D}$ is prenex. We now assume further that $\mathscr{D}$ includes no trivial simplications. In this case each formula of $\mathscr{D}$. occurring after the matrix change consists of a main prefix followed by a disjunction of what we will call main disjuncts. Any proper prenex subformula of any formula of $\mathscr{D}$ occurring after matrix change will be called a main disjunct of that formula. If $\bar{A}$ is an atomic part of some formula $\Delta_{1}$ occurring after the matrix change of $\mathscr{D}$ such that $\bar{A}$ is not part of a proper prenex subformula of $A_{1}$, then we define the main disjunct in which $\bar{A}$ occurs as follows. Let $\Delta_{2}$ the first subsequent formula of $\mathscr{D}$ in which the successor of $\bar{A}$ occurs in a proper prenex subformula. The main disjunct in which $\bar{A}$ occurs is that disjunct of the main alternation of $\Delta_{1}$ in which occur exactly those atomic parts of $\Delta_{1}$ whose successors in $\Delta_{2}$ occur in the proper prenex subformula of $\Delta_{2}$ in which the successor of $\bar{A}$ occurs. Here we assume $\Delta$ has at least one quantifier. Otherwise there are no applications of disassembling operations and the entire matrix of any formula of $\mathscr{D}$ after the matrix change is the only main disjunct of the formula. Any main disjunct of a formula of $\mathscr{D}$ is similar to $\Delta$. Suppose that $\Delta$ has no vacuous quantifier occurrences and that $\Delta_{1}$ is a formula occurring after all applica- 
tions of existential vacuous removal in $\mathscr{D}$. Then $\Delta_{1}$ has no vacuous quantifier occurrences and any main disjunct of $\Delta_{1}$ together with all quantifier occurrences of $A_{1}$ that apply nonvacuously to it is congruent to 4 .

LEMMA 1. Let $\Gamma$ be a prenex formula and $\Delta$ be a ND formula such that $\Delta$ is obtained from $\Gamma$ by an application of existential generalization, an application of existential vacuous removal, or by a series of applications of disassembling operations. Then $\Gamma$ is a ND formula.

Proof. By properties of the $L$-rules considered here it follows that $\Gamma$ is a prenex formula whose matrix is in disjunctive normal form. To show that $\Gamma$ is a $N D$ formula we consider together the cases of an application of existential generalization and an application of existential vacuous removal. For these cases $\Gamma$ and $\Delta$ are similar. If any two atomic parts of $\Gamma$, one positive and one negative, determine that $\Gamma$ is not a $N D$ formula, then their successors in $\Delta$ determine that $\Delta$ is not a $N D$ formula.

Next we assume that $\Delta$ is obtained from $\Gamma$ by a series of applications of disassembling operations. Suppose $\bar{A}_{1}$ and $\bar{B}_{2}$ are atomic parts, one positive and one negative, occurring in main disjuncts $\bar{\Gamma}_{1}$ and $\bar{\Gamma}_{2}$, respectively, of $\Gamma$. The formulas $\Gamma_{1}$ and $\Gamma_{2}$ of $\bar{\Gamma}_{1}$ and $\bar{\Gamma}_{2}$, respectively, are similar since they are both similar to $\Delta$. We let $\bar{B}_{1}$ be the atomic part of $\Gamma$ in $\bar{\Gamma}_{1}$ which corresponds to $\bar{B}_{2}$ in $\bar{\Gamma}_{2}$. We claim that if $\bar{A}_{1}$ and $\bar{B}_{2}$ determine that $\Gamma$ is not a $N D$ formula then so do $\bar{A}_{1}$ and $\bar{B}_{1}$. If $\bar{A}_{1}$ and $\bar{B}_{1}$ do not determine that $\Gamma$ is not a $N D$ formula and their atomic formulas $A_{1}$ and $B_{1}$, respectively, are the same atomic formula then the right most quantifier applying nonvacuously to this atomic formula is a universal quantifier. In this case either $A_{1}$ and $B_{2}$ are the same atomic formula (so that $\bar{A}_{1}$ and $\bar{B}_{2}$ would not determine that $\Gamma$ is not a $N D$ formula) or else they are atomic formulas which differ in the symbol occupying the individual variable place which contains the right most quantified individual variable (so that $\bar{A}_{1}$ and $\bar{B}_{2}$ again not determine that $\Gamma$ is not a $N D$ formula). That is, if $B_{1}$ and $B_{2}$ have this right most quantified individual variable in common then none of the quantifier occurrences applying nonvacuously to $\bar{B}_{1}$ and $\bar{B}_{2}$ can be imported before main disjuncts corresponding to $\bar{\Gamma}_{1}$ and $\bar{\Gamma}_{2}$ are simplicated together since this right most quantifier occurrence is to be imported first and, by restrictions imposed on importations, it would have the main disjuncts corresponding to both $\bar{\Gamma}_{1}$ and $\bar{\Gamma}_{2}$ in its scope after its importation. If $\bar{A}_{1}$ and $\bar{B}_{1}$ do not determine that $\Gamma$ is not a $N D$ formula and $A_{1}$ and 
$B_{1}$ are not the same atomic formula then $A_{1}$ and $B_{2}$ differ at least in the individual variables occupying the places at which $A_{1}$ and $B_{1}$ differ and therefore $\bar{A}_{1}$ and $\bar{B}_{2}$ do not determine that $\Gamma$ is not a $N D$ formula. Thus if there are two atomic parts of $\Gamma$ which determine that $\Gamma$ is not a $N D$ formula then there are two such atomic parts occurring in one occurrence of a main disjunct of $\Gamma$. But a main disjunct together with its nonvacuous quantifiers is congruent to $\Delta$ with its vacuous quantifiers deleted. It follows that $\Gamma$ is a $N D$ formula.

LEMMA 2. Let $Q N$ be a ND formula that is obtained from a prenex formula $P M$ by an application of existential generalization (by an application of existential vacuous removal). Let $C$ be a conjunction of signed atomic formulas the atomic formulas of which occur in $M$ such that $C \vDash M$. Then there exists a conjunction $D$ of signed atomic formulas the atomic formulas of which occur in $N$ such that $D \vDash N$ and $Q D$ can be obtained from $P C$ by applications of duplication and an existential generalization (by an application of existential vacuous removal).

Proof. By Lemma 1 it follows that $P M$ is a $N D$ formula. By the definition of the $L$-rule existential generalization (existential vacuous removal) $P M$ is similar to $Q N$. For the formulation in terms of an application of existential vacuous removal the conclusion of the lemma follows easily by letting $D=C$. Suppose that $Q N$ is obtained from $P M$ by an application of existential generalization. If an atomic formula that occurs in $C$ has both a positive and negative occurrence in $P M$ then either each occurrence of the atomic formula in $P M$ is identical with its successor in $Q N$ or all occurrences of the atomic formula differ from their successors in $Q N$ and all of the successors in $Q N$ of these occurrences are occurrences of the same atomic formula. Otherwise there would be positive occurrence and a negative occurrence of a predicate parameter in $Q N$ such that the occurrences are occurrences of atomic formulas which differ only in individual variables occupying individual variable places for which in at least one of the atomic parts the individual variable is existentially bound. This contradicts the hypothesis that $Q N$ is a $N D$ formula. The required formula $D$ is a conjunction (in the right order so necessary duplications can be applied) of signed atomic formulas which are the atomic formulas of successors of the atomic parts in $P M$ the atomic formulas of which occur in $C$.

We now present an example to show that the conditions given in the definition of a Negation Distinguished formula are necessary for our method. Let 


$$
\begin{aligned}
\Gamma_{1}= & \exists \exists b \exists c[[G b \wedge F a] \vee[H b \wedge \neg F a] \\
& \vee[G c \vee F a] \vee[H c \wedge \neg F a]], \\
\Gamma_{2}= & a \exists b \exists f \exists c[[G b \wedge F a] \vee[H b \wedge \neg F a] \\
\vee & {[G c \wedge F f] \vee[H c \wedge \neg F f]], } \\
\Gamma_{3}= & a \exists b[[G b \wedge F a] \vee[H b \wedge \neg F a]] \\
& \vee \exists a \exists b[[G b \wedge F a] \vee[H b \wedge \neg F a]],
\end{aligned}
$$

and

$$
\Delta=\mathrm{E} a \mathrm{E} b[[G b \wedge F a] \vee[H b \wedge \neg F a]] \text {. }
$$

Let $P, Q, M$, and $N$ be such that $P M=\Gamma_{1}$ and $Q N=\Gamma_{2}$ and let $C=G b \wedge H c$. Here $\Gamma_{2}$ is obtained from $\Gamma_{1}$ by an application of existential generalization and $C \vDash M$. However there is no conjunction $D$ of signed atomic formulas the atomic formulas of which occur in $N$ such that $D \vDash N$ and $P C \vDash Q D$. Thus to obtain a conclusion like that in Lemma 2 above we need to require that $Q N$ has no occurrences of subformulas like $F a$ and $7 F f$ here formed with atomic formulas that differ only in the individual variable occupying an individual variable place containing an existentially bound individual variable in at least one of the atomic parts. In our applications we will want to assume that a concluding formula $\Delta$ of a symmetric $L$-deduction is a $N D$ formula and from this conclude by Lemma 1 that formulas to which existential generalizations are applied in the $L$-deduction are $N D$ formulas so that a conclusion like that of Lemma 2 can be obtained for the results of these existential generalizations. Our example also shows that in order to prove Lemma 1 we need the condition in case (1) of the definition of a Negation Distinguished formula requiring that a right most quantifier be a universal quantifier. Here $\Gamma_{3}$ is obtained from $\Gamma_{2}$ by applications of existential importation and $\Delta$ is obtained from $\Gamma_{3}$ by a simplication. $\Delta$ fails to be a $N D$ formula only because of the requirement that certain quantifiers are to be universal quantifiers. However $\Gamma_{2}$ fails to be a $N D$ formula because of other conditions in the definition of a $N D$ formula which are important in obtaining the conclusion of Lemma 2 .

LEMma $3^{1}$. Let $\Gamma$ be a prenex formula with a valid matrix and let $\Delta$ be a ND formula such that $\Delta$ is obtained from $\Gamma$ by an application of existential generalization, an application of existential vacuous removal, or by a series of application of disassembling operations. Then the matrix of $\Delta$ is valid.

1 For Lemma 3 and Theorem 1 condition (1) of the definition of $N D$ formula is unnecessary. 
Proof. If $\Delta$ is obtained from $\Gamma$ by an application of existential vacuous removal then the matrix of $\Delta$ is the same as that of $\Gamma$ and so it is valid. Suppose $\Delta$ is obtained from $\Gamma$ by an application of existential generalization. Since $\Delta$ is a $N D$ formula it follows that if an atomic formula has both a positive and a negative occurrence in $\Gamma$ then all successors in $\Delta$ of any occurrences of the atomic formula in $\Gamma$ are occurrences of the same atomic formula. The matrix of $\Delta$ is obtained from the matrix of $\Gamma$ by replacing occurrences of atomic formulas with other atomic formulas in such a way that an atomic formula having both a positive and negative occurrence in $\Gamma$ is replaced in all of its occurrences by the same atomic formula. It follows that the matrix of $\Delta$ is valid. Finally suppose $\Delta$ is obtained from $\Gamma$ by a series of applications of disassembling operations. In this case some of the intermediate formulas may not be prenex, so we let the matrix of any formula be the quantifier free formula obtained by deleting all of its quantifiers. We see that each of the disassembling operations preserves the property of a formula of having a valid matrix. Thus for all three cases we conclude that the matrix of $\Delta$ is valid.

THEOREM 1. A ND formula is valid if and only if its matrix is valid.

Proof. Suppose $\Delta$ is a $N D$ formula that is valid and let $A$ be any propositional parameter that does not occur in $\Delta$. Then $A \vDash A$, so there exists a symmetric $L$-deduction, say $\mathscr{D}$, of $\Delta$ from $A$. By properties of $L$-rules that can be applied before the matrix change in a symmetric $L$-deduction it follows that the matrix of the formula to which the operation matrix change is applied in $\mathscr{D}$ is a conjunction of occurrences of $A$. By properties of $L$-rules that can be applied after the matrix change in a symmetric $L$-deduction it follows that the predicate parameter $A$ that does not occur in $\Delta$ also does not occur in the formula resulting from the application of matrix change. It follows that the matrix of the formula resulting from the matrix change in $\mathscr{D}$ is valid. By Lemma 3 it follows that the matrix of $\Delta$ is valid. Conversely, if we assume that the matrix of a $N D$ formula is valid then it follows that the formula is valid.

LEMMA 4. Let $\Gamma$ and $\Delta$ be prenex formulas such that $\Delta$ is a ND formula, the matrix of $\Delta$ is not valid, the matrix of $\Gamma$ is a conjunction of signed atomic fomulas, and $\Gamma \rightarrow \Delta$ is valid. Then there exists an L-deduction $\left\langle\Gamma, \cdots, Q^{\prime} C^{\prime}, Q^{\prime} N^{\prime}, \cdots, \Delta\right\rangle$ such that $\left\langle\Gamma, \cdots, Q^{\prime}, C^{\prime}\right\rangle$ is a symmetric L-deduction in which no disassembling operations occur, $\left\langle Q^{\prime} N^{\prime}, \cdots, \Delta\right\rangle$ is an L-deduction in which only dis- 
assembling operations occur, and $C^{\prime}$ is a conjunction of signed atomic formulas the atomic formulas of which occur in $N^{\prime}$.

Proof. Let $\Gamma$ and $\Delta$ be as prescribed. Then there exists a symmetric $L$-deduction, say $\mathscr{D}$, of $\Delta$ from $\Gamma$. Let $P M$ be the formula resulting from the application of matrix change in $\mathscr{D}$. By successive applications of Lemma 1 it follows that $P M$ and all subsequent prenex formulas of $\mathscr{D}$ are $N D$ formulas. Let $Q^{\prime} N^{\prime}$ be the formula occurring just before disassembling operations in $\mathscr{D}$. The existence of the conjunction $C^{\prime}$ of signed atomic formulas the atomic formulas of which occur in $N^{\prime}$ such that $C^{\prime} \vDash N^{\prime}$ is established by successive applications of Lemma 2 to the two line subdeductions of $\mathscr{D}$ consisting of successive formulas occurring between $P M$ and $Q^{\prime} N^{\prime}$. For the first application of Lemma 2 the matrix $M$ of $P M$ is taken as the conjunction of signed atomic formulas required in the hypothesis of Lemma 2. Let $\mathscr{E}$ be the $L$-deduction obtained by continuing on from the subdeduction of $\mathscr{D}$ of $P M$ from $\Gamma$ by piecing together two line deductions obtained from the conclusions of the applications of Lemma 2. The symmetric $L$-deduction $\left\langle\Gamma, \cdots, Q^{\prime} C^{\prime}\right\rangle$ required in this lemma is obtained from $\mathscr{E}$ by replacing any occurrences of duplication after the matrix change with an obvious modification of the matrix change. Then $\left\langle\Gamma, \cdots, Q^{\prime} C^{\prime}, Q^{\prime} N^{\prime}, \cdots, \Delta\right\rangle$ is obtained from $\left\langle\Gamma, \cdots, Q^{\prime} C^{\prime}\right\rangle$ by continuing on with the subdeduction of $\mathscr{D}$ of $\Delta$ from $Q^{\prime} N^{\prime}$.

3. Modified symmetric $\boldsymbol{L}$-deductions. Now we consider a particular kind of $L$-deduction that arises from applications of Lemma 4 above. For any prenex formulas $\Gamma$ and $\Delta$ whose matrices are conjunctions of signed atomic formulas, a modified symmetric $L$-deduction of $\Delta$ from $\Gamma$ is an $L$-deduction $\mathscr{D}$ of $\Delta$ from $\Gamma$ satisfying the following conditions :

(1) There is a prenex formula, say $A_{1}$, whose matrix is a conjunction of signed atomic formulas and such that the subdeduction of $\mathscr{D}$ of $\Delta_{1}$ from $\Gamma$ satisfies all of the conditions of being a symmetric $L$-deduction in which no disassembling operations occur except that vacuous existential generalizations may occur immediately before universal instantiations.

(2) The subdeduction of $\mathscr{D}$ of $\Delta$ from $\Delta_{1}$ consists of, first an application of matrix change the effect of which is the deletion of zero or more but not all of the conjuncts of the matrix of $\Delta_{1}$, then zero or more applications of existential vacuous removal, and finally zero or more applications of universal instantiation for which the universal quantifier occurrences to be instantiated are vaçous, 
Let $\mathscr{D}$ be a modified symmetric $L$-deduction of $\Delta$ from $\Gamma$ where $\Delta$ and $\Gamma$ are prenex formulas whose matrices are conjunctions of signed atomic formulas. Each formula of $\mathscr{D}$ occurring before the first application of matrix change consists of a prefix followed by a conjunction, each conjunct of which is called a main conjunct and is an occurrence of a prenex formula similar to $\Gamma$. Each formula occurring: after the first application of matrix change in $\mathscr{D}$ is a prenex formula the matrix of which is a conjunction of signed atomic formula. We will call each of these conjuncts a main conjunct of the formula in which it occurs. If $\Gamma_{i}$ and $\Gamma_{i+1}$ are two successive formulas of $\mathscr{D}$ and $\left\{\bar{C}_{1}, \cdots, \bar{C}_{h}\right\}$ is any set of main conjuncts of $\Gamma_{i}$ then we define the corresponding set of main conjuncts of $\Gamma_{i+1}$ as follows. If $\Gamma_{i}$ and $\Gamma_{i+1}$ occur before the first application of matrix change in $\mathscr{D}$ then the corresponding set of main conjuncts of $\Gamma_{i+1}$ includes those main conjuncts of $\Gamma_{i+1}$ in which occur an atomic part of $\Gamma_{i+1}$ the predecessor of which in $\Gamma_{i}$ occurs in some $\bar{C}_{i}, i=1, \cdots, h$. If $\Gamma_{i+1}$ is obtained from $\Gamma_{i}$ by an application of matrix change in $\mathscr{D}$ then $\Gamma_{i}$ and $\Gamma_{i+1}$ are prenex formulas the matrices of which are conjunctions of signed atomic formulas and the corresponding set of main conjuncts of $\Gamma_{i+1}$ includes any conjuncts of the matrix of $\Gamma_{i+1}$ that must be deleted to obtain a formula that is a consequence of the formula obtained from the matrix of $\Gamma_{i}$ by deleting $\bar{C}_{1}, \cdots, \bar{C}_{h}$. If $\Gamma_{i+1}$ occurs after the first application of matrix change in $\mathscr{D}$ and is obtained from $\Gamma_{i}$ by an application of a quantifier rule then $\Gamma_{i}$ and $\Gamma_{i+1}$ are similar prenex formulas and the corresponding set of main conjuncts of $\Gamma_{i+1}$ includes those in the same relative position as $\bar{C}_{1}, \cdots, \bar{C}_{h}$. Let $\Gamma_{i}$ and $\Gamma_{i+1}$ be successive formulas of $\mathscr{D}$, let $\Gamma_{i}^{1}$ be a formula obtained by deletion of a set of main conjuncts of $\Gamma_{i}$ and let $\Gamma_{i+1}^{1}$ be the formula obtained from $\Gamma_{i+1}$ by deleting the corresponding set of main conjuncts in $\Gamma_{i+1}$ and also by deleting the quantifier occurrence just introduced into the main prefix of $\Gamma_{i+1}$ in case $\Gamma_{i+1}$ is obtained from $\Gamma_{i}$ by an exportation of a quantifier from a main conjunct occurrence of $\Gamma_{i}$ that was deleted to obtain $\Gamma_{i}^{1}$. By the definitions of the $L$-rules it follows that $\Gamma_{i+1}^{1}$ is identical with $\Gamma_{i}^{1}$ or $\Gamma_{i+1}^{1}$ is obtained from $\Gamma_{i}^{1}$ by an application of the same $L$-rule which is applied in $\mathscr{D}$ to obtain $\Gamma_{i+1}$ from $\Gamma_{i}$. A modified symmetric $L$-deduction $\mathscr{D}^{\prime}$ will be said to be obtained from $\mathscr{D}$ by duplicate deletion in case $\mathscr{D}^{\prime}$ is obtained from $\mathscr{D}$ in the following way. There is a formula, say $\Gamma_{i}$, to which duplication is applied in $\mathscr{D}$. One of the two identical main conjuncts introduced into $\Gamma_{i+1}$ by the application of duplication is deleted and the corresponding sets of main conjuncts of the successive formulas of $\mathscr{D}$ are deleted. Quantifier occurrences that were introduced into the main prefix of formulas of $\mathscr{D}$ by exportation from a main conjunct that is deleted are also deleted 
except that any which later apply nonvacuously to other atomic parts of the formula because of universal instantiations are introduced by universal vacuous introduction or existential generalization, which happens to be vacuous, at the appropriate place in the prefix.

LEMMA 5. There exists an effective procedure for deciding, for any prenex formulas $\Gamma$ and $\Delta$ whose matrices are conjunctions of signed atomic formulas, whether there exists a modified symmetric $L$-deduction of $\Delta$ from $\Gamma$.

Proof. Let $n$ be the number of conjuncts in the matrix of 4 . If we have a modified symmetric $L$-deduction of $\Delta$ from $\Gamma$, then by a succession of applications of duplicate deletion we can obtain one in which no more than $n-1$ duplications occur. Here we may delete a main conjunct introduced in an application of duplication unless the final formula of the resulting deduction does not have a main conjunct corresponding to one of the $n$ main conjuncts of 4 . Let $h$ be the number of occurrences of universal quantifiers and $k$ the number of occurrences of existential quantifiers in $\Delta$. If we have a modified symmetric $L$-deduction of $\Delta$ from $\Gamma$, then we may delete from it all but $\leqq h$ applications of universal vacuous introduction together with the corresponding applications of universal (vacuous) instantiation and all but $\leqq k$ applications of existential generalization together with the corresponding applications of existential vacuous removal. To determine whether there exists a modified symmetric $L$-deduction of $\Delta$ from $\Gamma$ we consider each member of a maximal set of noncongruent modified symmetric $L$-deductions from $\Gamma$ which include no more than $n$ applications of duplication, $h$ applications of universal vacuous introduction, $k$ applications of existential generalization and in which the matrix of the formula resulting from the last application of matrix change is similar to $\Delta$.

4. Disassembling operations. In this section we provide a procedure for determining the existence of $L$-deductions in which only disassembling operations occur and then we combine the procedure with earlier results to obtain our major theorem.

For each $Q$ and $N$ let $\mid \bar{N} Q$ be the results of deleting from $Q$ all quantifier occurrences which in $Q N$ would be vacuous. For prenex formulas $Q N$ and $Q^{\prime} N^{\prime}=Q^{\prime}\left[N_{1} \vee \cdots \vee N_{e}\right]$ in which there are no vacuous quantifier occurrences we say that $Q N$ is a $D$-consequent of $Q^{\prime} N^{\prime}$ and write $Q^{\prime} N^{\prime} \vDash_{D} Q N$ in case each $\mid \bar{N}_{i} Q^{\prime} N_{i}$ is congruent to $Q N$ and for any $i \neq j$ there are $Q_{1}, Q_{2}, Q_{3}$ with $Q_{1}$ possibly empty such that $\left|\bar{N}_{i} Q^{\prime}=Q_{1} Q_{2},\right| \overline{N_{j}} Q^{\prime}=Q_{1} Q_{3}$ and $\left(Q_{1}\right), Q_{2}$, and $Q_{3}$ have no variable 
in common. In this case we call the occurrences of the disjuncts $N_{1}, \cdots, N_{e}$ main disjuncts of $Q^{\prime} N^{\prime}$ and of $N^{\prime}$. The following Lemma is an immediate consequence of the definitions of the disassembling operations.

LEMMA 6. The following two conditions are equivalent:

(1) $Q N$ and $Q^{\prime} N^{\prime}$ are prenex formulas such that $Q N$ has no vacuous quantifier occurrences and is obtained from $Q^{\prime} N^{\prime}$ by a sequence of applications of disassembling operations in which no trivial simplications occur;

(2) $Q^{\prime} N^{\prime}$ has the form $Q^{\prime} N^{\prime}=Q^{\prime}\left[N_{1} \vee \cdots \vee N_{e}\right]$ with no vacuous quantifier occurrences and $Q^{\prime} N^{\prime} \vDash{ }_{D} Q N$.

LEMmA 7. Let $\theta, \sigma, \phi$ be distinct propositional variables, let $H(\theta, \sigma)$ and $J(\sigma)$ be propositional formulas containing only the variables indicated, and let $H(\phi, \sigma)$ be the result of substituting $\phi$ for $\theta$ in $H(\theta, \sigma)$. Then $H(\theta, \sigma) \wedge H(\phi, \sigma) \vDash J(\sigma)$ implies that $H(\theta, \sigma) \vDash J(\sigma)$.

Proof. Suppose the conclusion is false. Then it is easy to describe an assignment of truth values to all propositional variables in such a way as to demonstrate that the assumed logical implication does not hold.

Let $p(n)$ be the number of partitions of $n$ for any positive integer $n$ (vid. page 273 of [3]). For any nonnegative integer $h$ let $m_{h}(i)$ be defined for positive integers $i$ by the equations $m_{h}(1)=1$ and

$$
m_{h}(i+1)=m_{h}(i) p\left(m_{h}(i)\right)^{i m_{h(i)}} 3^{m_{h(i) h}} \text {. }
$$

For any prenex formula $Q N$ with $k$ bound individual variables and $h$ atomic formulas in which bound individual variables occur we let $g(Q N)=m_{h}(k+1)$. We observe that $g$ is an effectively calculable function.

Lemma 8. Let $Q N$ and $Q^{\prime} N^{\prime}=Q^{\prime}\left[N_{1} \vee \cdots \vee N_{e}\right]$ be prenex formulas with no vacuous quantifier occurrences such that $Q^{\prime} N^{\prime} \vDash_{D} Q N$. Let $C^{\prime}$ be any conjunction of signed atomic formulas such that all atomic formulas of $C^{\prime}$ occur in $N^{\prime}$ and such that $C^{\prime} \vDash N^{\prime}$. Then there is an $N^{\prime \prime}$ obtained from $N^{\prime}$ by deleting all but $\leqq g(Q N)$ main disjuncts (of $N^{\prime}$ ) such that $C^{\prime} \vDash N^{\prime \prime}$.

Proof. Let $Q N, Q^{\prime} N^{\prime}$ and $C^{\prime}$ be as prescribed and assume $Q N$ has $k$ bound individual variables and $h$ atomic formulas in which bound individual variables occur. Let $N^{\prime \prime}$ be any matrix obtained from $N^{\prime}$ by deleting zero or more main disjuncts such that $C^{\prime} \vDash N^{\prime \prime}$ and such 
that if any additional main disjuncts are deleted the result is either the empty matrix or is not a consequence of $C^{\prime}$. Thus $Q^{\prime} N^{\prime \prime}$ has a minimum number of the main disjuncts of $Q^{\prime} N^{\prime}$. We will show that this minimum number of main disjuncts of $Q^{\prime} N^{\prime}$ that remain in $Q^{\prime} N^{\prime \prime}$ is $\leqq g(Q N)$.

For any bound individual variable in $Q^{\prime} N^{\prime}$ we define its order to be a positive integer determined as follows. Select any main disjunct of $Q^{\prime} N^{\prime}$ in which the individual variable occurs, delete from $Q^{\prime} N^{\prime}$ all quantifier occurrences that bind no individual variable occurrences in this main disjunct, and then assign as the order of the individual variable the number of the position of its binding quantifier occurrence counting from right to left among the remaining quantifier occurrences. From the definition of $F_{D}$ it follows that this definition is independent of the main disjunct selected to determine the order for bound individual variables that occur in more than one main disjunct. For any atomic formula occurring in $Q^{\prime} N^{\prime}$ in which occurs a bound individual variable we define the order of the atomic formula to be the order of the bound individual variable of least order that occurs in it. Let $h_{i}$ be the number of atomic formulas of order $i$ that occur in any one main disjunct of $Q^{\prime} N^{\prime}$ for $i=1, \cdots, k$. Since each $\mid \overline{N_{i}} Q^{\prime} N_{i}$ is congruent to $Q N$ it follows that $h=h_{1}+\cdots+h_{k}$. Let $\rho_{i}(i=1, \cdots, k)$ be the equivalence relation defined on the set of main disjuncts of $Q^{\prime} N^{\prime}$ as follows. For main disjuncts $N_{u}$ and $N_{v}, N_{u}$ is $\rho_{i}$ related to $N_{v}$ if and only if the bound individual variables of order $i$ are the same in $N_{u}$ and $N_{v}$. We let $\rho_{k+1}$ be the universal relation on the set of main disjuncts. By properties of $\vDash_{D}$ it follows that if two main disjuncts have a bound individual variable in common then any bound individual variable of greater order in one of the two main disjuncts is identical with the bound individual variable of the same greater order in the other main disjunct. Thus if $1 \leqq i \leqq j \leqq k+1$ then $\rho_{i} \leqq \rho_{j}$. Also, if $1 \leqq i \leqq k$ and if $N_{u}$ and $N_{v}$ are two main disjuncts that are not $\rho_{i}$ related, then any atomic formula occurring in $N_{u}$ that is of order $\leqq i$ does not occur in $N_{v}$. Let $\bar{\rho}_{i}$ be the restriction of $\rho_{i}$ to the set of main disjuncts of $N^{\prime}$ that are not deleted to obtained $N^{\prime \prime}$. Let $\bar{m}(i)$ be the maximum number of main disjuncts that occur in any $\bar{\rho}_{i}$ class for $i=1, \cdots, k+1$. Using the fact that $Q^{\prime} N^{\prime \prime}$ has a minimum number of main disjuncts we apply a generalization of Lemma 7 to obtain an upper bound for $\bar{m}(i+1)$ in terms of $\bar{m}(i)$ for $i=1, \cdots, k$. We first assume that we know $\bar{m}(i)$ and from this determine an upper bound $b(i)$ for the number of $\bar{\rho}_{i}$ classes included in any one $\bar{\rho}_{i+1}$ class for $1 \leqq i \leqq k$. For a given $\bar{\rho}_{i+1}$ class $\mathscr{C}_{i+1}$ let $\mathscr{T}$ be the set of ordered pairs $\langle C, F\rangle$ where $F$ is the disjunction of all main disjuncts in a $\bar{\rho}_{i}$ class which is included in $\mathscr{C}_{i+1}$ and $C$ is the subconjunction of $C^{\prime}$ the conjuncts of which are 
formed with atomic formulas that occur in $F$. Here we identify two disjunctions $F$ that differ only in the order of their disjuncts so that $\mathscr{T}$ contains exactly one member for each $\bar{\rho}_{i}$ subclass of $\mathscr{C}_{i+1}$. We will show that no two members of $\mathscr{T}$ are congruent. Otherwise, suppose $\left\langle C_{1}, F_{1}\right\rangle$ and $\left\langle C_{2}, F_{2}\right\rangle$ are two members of $\mathscr{T}$ such that (after perhaps permutations of disjuncts and conjuncts) $C_{1}$ and $C_{2}$ are similar, $F_{1}$ and $F_{2}$ are similar, and such that $\left\langle C_{1}, F_{1}\right\rangle$ can be obtained from $\left\langle C_{2}, F_{2}\right\rangle$ by alphabetic changes of individual variables in such a way that the same individual variable occurs at two places in $\left\langle C_{1}, F_{1}\right\rangle$ if and only if the individual variable occurring at the two corresponding places in $\left\langle C_{2}, F_{2}\right\rangle$ are the same. Let $D$ be the subconjunction of $C^{\prime}$ including all conjuncts the atomic formulas of which do not occur in $C_{1}$ or $C_{2}$ and let $G$ be the disjunction of all main disjuncts of $N^{\prime \prime}$ that do not occur in $F_{1}$ or $F_{2}$. Here the empty conjunct is taken as the logical constant truth and the empty disjunction is taken as the logical constant falsity. The assumption that $C^{\prime} \vDash N^{\prime \prime}$ is equivalent to the condition that $C_{1} \wedge \neg F_{1} \wedge C_{2} \wedge \neg F_{2} \vDash \neg D \vee G$. In the statement of Lemma 7 replace $H(\theta, \sigma)$ by $C_{1} \wedge \neg F_{1}, H(\phi, \sigma)$ by $C_{2} \wedge \neg F_{2}$ and $J(\sigma)$ by $\neg D \vee G$. Then, with $\left\langle C_{1}, F_{1}\right\rangle$ and $\left\langle C_{2}, F_{2}\right\rangle$ as indicated above, the generalization of Lemma 7 obtained by replacing each of $\theta, \phi$ and $\sigma$ with a set of propositional variables (here taken as the atomic formulas of $N^{\prime \prime}$ ) would show that $Q^{\prime} N^{\prime \prime}$ does not have a minimum number of main disjuncts of $Q^{\prime} N^{\prime}$. Here any common atomic formulas of $F_{1}$ and $F_{2}$ appear in corresponding positions in $F_{1}$ and $F_{2}$ since any two main disjuncts of $Q^{\prime} N^{\prime}$ have an individual variable in common if and only if it is the individual variable of the same order in both main disjuncts. Thus no two members of $\mathscr{T}$ are congruent.

To determine an uppor bound $b(i)$ for the number of elements in $\mathscr{T}$ (and thus for the number of $\bar{\rho}_{i}$ classes included in $\mathscr{C}_{i+1}$ ) we observe that the number of non-congruent formulas $F$ that are the disjunctions of the main disjuncts in a $\bar{\rho}_{i}$ subclass of $\mathscr{C}_{i+1}$ is $\leqq p(\bar{m}(i))^{i m(i)}$. Suppose $F_{1}$ is the disjunction of all main disjuncts in a $\bar{\rho}_{i}$ class $\mathscr{C}_{i}^{1}$ and $F_{2}$ is the disjunction of all main disjuncts in another $\bar{\rho}_{i}$ class $\mathscr{C}_{i}^{2}$. Then $F_{1}$ and $F_{2}$ are congruent if and only if $\mathscr{C}_{i}^{1}$ and $\mathscr{C}_{i}^{2}$ are isomorphic with respect to the relations $\bar{\rho}_{u}$ for $u<i$ (that is, if and only if $\mathscr{C}_{i}^{1}$ and $\mathscr{C}_{i}^{2}$ include the same number of $\bar{\rho}_{i-1}$ classes which can be paired up in such a way that a $\bar{\rho}_{i-1}$ subclass of $\mathscr{C}_{i}^{\prime}$ includes the same number of $\bar{\rho}_{i-2}$ classes as the corresponding $\bar{\rho}_{i-1}$ subclass of $\mathscr{C}_{i}^{2}$ etc.) There are $\leqq p(\bar{m}(i))$ ways of partitioning the set of $\leqq \bar{m}(i)$ main disjuncts in a $\bar{\rho}_{i}$ class into distinct $\bar{\rho}_{i-1}$ classes. If $u \leqq i$ then $\bar{m}(u) \leqq \bar{m}(i)$ and the number of $\bar{\rho}_{u}$ classes included in a $\bar{\rho}_{i}$ class is $\leqq \bar{m}(i)$. It follows that the number of ways of first partitioning the set of main disjuncts in a $\bar{\rho}_{i}$ class into $\bar{\rho}_{i-1}$ classes, then partitioning these $\bar{\rho}_{i-1}$ classes into 
$\bar{\rho}_{i-2}$ classes, $\cdots$ and then partitioning these $\bar{\rho}_{2}$ classes into $\bar{\rho}_{1}$ classes, is $\leqq p(\bar{m}(i))^{i \bar{m}(i)}$. To take account of non-congruent elements $\left\langle C_{1}, F_{1}\right\rangle$, $\left\langle C_{2}, F_{2}\right\rangle \in \mathscr{T}$ where $F_{1}$ and $F_{2}$ are congruent, we observe that there are $\leqq \bar{m}(i)\left(h_{1}+\cdots+h_{i}\right) \leqq \bar{m}(i) h$ atomic formulas of order $\leqq i$ occurring in any formula $F$ that is a disjunction of no more than $\bar{m}(i)$ main disjuncts. Each of these $\leqq \bar{m}(i) h$ atomic formulas may either not occur, occur positively, or occur negatively in such a conjunction $C$. We need not consider the case of an atomic formula occurring both positively and negatively in $C$ since, for our applications, $C$ is a subconjunction of the conjunction $C^{\prime}$ appearing in the statement of the lemma and the conclusion of the lemma follows immediately if $C^{\prime}$ is a contradiction. Thus for the upper bound $b(i)$ we may take $p(\bar{m}(i))^{i \bar{m}(i)} 3^{h \bar{m}(i)}$. By the definition of $\bar{m}(i)$, this implies that

$$
\bar{m}(i+1) \leqq \bar{m}(i) b(i)=\bar{m}(i) p(\bar{m}(i))^{i \bar{m}(i)} 3^{h \bar{m}(i)},
$$

for $1 \leqq i<k$. The number of main disjuncts of $Q^{\prime} N^{\prime}$ that remain in $Q^{\prime} N^{\prime \prime}$ is $\leqq \bar{m}(k) b(k)$. The formulas of any two main disjuncts that are $\rho_{1}$ related are identical so $\bar{m}(1)=1$. From the inequalities established above relating $\bar{m}(i+1)$ and $\bar{m}(i)$ and from the definition of $m_{h}(i)$ given above it follows that $\bar{m}(k) b(k) \leqq g(Q N)$.

THEOREM 2. There exists an effective procedure for deciding for any prenex formula $\Gamma$ the matrix of which is a conjunction of signed atomic formulas and any ND formula $\Delta$ whether $\Gamma \rightarrow \Delta$ is valid.

Proof. Let $\Gamma$ and $\Delta$ be as prescribed and for convenience we also assume $\Delta$ has no vacuous quantifier occurrences. First determine whether the matrix of $\Delta$ is valid. If it is then $\Gamma \rightarrow \Delta$ is valid. If not proceed as follows. First, determine the effectively calculable number $g(\Delta)$. Second, determine a set $\mathscr{S}$ of ordered pairs of formulas $\langle C, Q N\rangle$ with the following properties:

(1) For any element $\langle C, Q N\rangle$ of $\mathscr{S}, C$ is a conjunction of signed atomic formulas the atomic formulas of which occur in $N, C$ has no more than two occurrences of any one atomic formula, and $C \vDash N$.

(2) For any element $\langle C, Q N\rangle$ of $\mathscr{S}, \Delta$ can be obtained from $Q N$ by applications of disassembling operations and $N$ has no more than $g(\Delta)$ main disjuncts (relative to $\Delta$ ).

(3) There are no two members of $\mathscr{S}$ of the form $\left\langle C^{\prime}, Q^{\prime} N^{\prime}\right\rangle$ and $\langle C, Q N\rangle$ such that $C$ is similar $C^{\prime}$ and $Q N$ is congruent to $Q^{\prime} N^{\prime}$ and such that $\left\langle C^{\prime}, Q^{\prime} N^{\prime}\right\rangle$ is obtained from $\langle C, Q N\rangle$ by alphabetic changes of individual variables in such a way that the same individual variable occurs at two places in $\left\langle C^{\prime}, Q^{\prime} N^{\prime}\right\rangle$ if and only if the individual variables occurring at the two corresponding places in $\langle C, Q N\rangle$ are the same.

(4) $\mathscr{S}$ is a maximal set with respect to set inclusion satisfying 
conditions (1), (2) and (3) above. It is easy to see that there is an effective procedure for determining such a set $\mathscr{S}$. If a formula $Q N$ occurs in a element $\langle C, Q N\rangle$ of $\mathscr{S}$ then it must have no vacuous quantifier occurrences and its matrix must be a disjunction of $1,2, \cdots$, or $g(\Delta)$ matrices each similar to the matrix of $\Delta$. One can first obtain a maximal set $\mathscr{C}$ of pairs of formulas $\langle C, Q N\rangle$ satisfying these two conditions and conditions (1) and (3) above. Then the required set $\mathscr{S}$ can be obtained from $\mathscr{Q}$ by deleting those members of $\mathscr{U}$ for which condition (2) is not satisfied. One can effectively decide whether $\Delta$ can be obtained from a given prenex formula $Q N$ by applications of disassembling operations since in disassembling operations quantifiers must be imported in their right to left order. Third, decide for each element $\langle C, Q N\rangle$ of $\mathscr{S}$ whether there is a modified symmetric $L$-deduction of $Q C$ from $\Gamma$. By Lemma 5 this step is effective. We conclude that $\Gamma \rightarrow \Delta$ is valid if and only if the answer is yes in step three for at least one member of $\mathscr{S}$.

It is easy to see that if the answer is yes in part three for one member of $\mathscr{S}$, say for $\left\langle C_{1}, Q_{1} N_{1}\right\rangle$, then $\Gamma \rightarrow \Delta$ is valid. In this case $\Gamma \vDash Q_{1} C_{1}$, and $C_{1} \vDash N_{1}$ so $Q_{1} C_{1} \vDash Q_{1} N_{1}$. Hence $\Gamma \vDash Q_{1} N_{1}$. By the definition of $\mathscr{S}, Q_{1} N_{1} \vDash \Delta$ so $\Gamma \vDash \Delta$. That is, $\Gamma \rightarrow \Delta$ is valid.

Conversely, suppose that $\Gamma \rightarrow \Delta$ is valid. Then the hypotheses of Lemma 4 are satisfied for $\Gamma$ and $\Delta$. Let $\left\langle\Gamma, \cdots, Q^{\prime} C^{\prime}, Q^{\prime} N^{\prime}, \cdots, \Delta\right\rangle$ be the $L$-deduction whose existence is asserted by the application of Lemma 4 to $\Gamma$ and 4 . By Lemma 8 and 6 we may delete all but $\leqq g(\Delta)$ main disjuncts of $Q^{\prime} N^{\prime}$ and all quantifier occurrences thereby made vacuous to obtain a formula $Q^{\prime \prime} N^{\prime \prime}$ such that $\Delta$ can be obtained from $Q^{\prime \prime} N^{\prime \prime}$ by applications of disassembling operations and such that if $C^{\prime \prime}$ is the subconjunction of $C^{\prime}$ formed with conjuncts the atomic formulas of which occur in $Q^{\prime \prime} N^{\prime \prime}$ then $C^{\prime \prime} \vDash N^{\prime \prime}$. Thus $\left\langle C^{\prime \prime}, Q^{\prime \prime} N^{\prime \prime}\right\rangle$ or an ordered pair congruent to it is in $\mathscr{S}$. The $L$-deduction $\left\langle\Gamma, \cdots, Q^{\prime} C^{\prime}\right\rangle$ can be extended to a modified symmetric $L$-deduction $\left\langle\Gamma, \cdots, Q^{\prime} C^{\prime}, \cdots, Q^{\prime \prime} C^{\prime \prime}\right\rangle$ so that the answer is yes in step three for one member of $\mathscr{S}$.

\section{BIBLIOGRAPHY}

1. A. Church, Introduction to Mathematical Logic, Vol. I. Princeton University Press, Princeton, 1956.

2. W. Craig, Linear reasoning. A new form of the Herbrand-Gentzen theorem, J. Symbolic Logic, 22 (1957), 250-268.

3. G. H. Hardy and E. M. Wright, An Introduction to the Theory of Numbers, Third edition, Oxford University Press, London, 1954.

4. J. Herbrand, Sur le probleme fondamental de la logique mathematique, Comptes Rendus Des Seances De La Societe Des Sciences Et Des Lettres De Varsovie, Class III, 24 (1931) 12-56.

UNIVERSITY OF CALIFORNIA, DAVIS 



\title{
PACIFIC JOURNAL OF MATHEMATICS
}

\author{
EDITORS
}

\author{
RoBerit OsSERman \\ Stanford University \\ Stanford, California
University of Washington
Seattle 5 , Washington \\ M. G. Arsove
}

\author{
J. DugundjI \\ University of Southern California
}

Los Angeles 7, California

Lowell J. Paige

University of California

Los Angeles 24, California

\section{ASSOCIATE EDITORS}
E. F. BECKENBACH
B. H. NeumanN
F. WOLF
K. YOSIDA

\section{SUPPORTING INSTITUTIONS}

\author{
UNIVERSITY OF BRITISH COLUMBIA \\ CALIFORNIA INSTITUTE OF TECHNOLOGY \\ UNIVERSITY OF CALIFORNIA \\ MONTANA STATE UNIVERSITY \\ UNIVERSITY OF NEVADA \\ NEW MEXICO STATE UNIVERSITY \\ OREGON STATE UNIVERSITY \\ UNIVERSITY OF OREGON \\ OSAKA UNIVERSITY \\ UNIVERSITY OF SOUTHERN CALIFORNIA
}

\author{
STANFORD UNIVERSITY \\ UNIVERSITY OF TOKYO \\ UNIVERSITY OF UTAH \\ WASHINGTON STATE UNIVERSITY \\ UNIVERSITY OF WASHINGTON \\ * * * * \\ AMERICAN MATHEMATICAL SOCIETY \\ CALIFORNIA RESEARCH CORPORATION \\ SPACE TECHNOLOGY LABORATORIES \\ NAVAL ORDNANCE TEST STATION
}

Mathematical papers intended for publication in the Pacific Journal of Mathematics should by typewritten (double spaced), and on submission, must be accompanied by a separate author's résumé. Manuscripts may be sent to any one of the four editors. All other communications to the editors should be addressed to the managing editor, L. J. Paige at the University of California, Los Angeles 24, California.

50 reprints per author of each article are furnished free of charge; additional copies may be obtained at cost in multiples of 50 .

The Pacific Journal of Mathematics is published quarterly, in March, June, September, and December. Effective with Volume 13 the price per volume (4 numbers) is $\$ 18.00$; single issues, $\$ 5.00$. Special price for current issues to individual faculty members of supporting institutions and to individual members of the American Mathematical Society: $\$ 8.00$ per volume; single issues $\$ 2.50$. Back numbers are available.

Subscriptions, orders for back numbers, and changes of address should be sent to Pacific Journal of Mathematics, 103 Highland Boulevard, Berkeley 8, California.

Printed at Kokusai Bunken Insatsusha (International Academic Printing Co., Ltd.), No. 6, 2-chome, Fujimi-cho, Chiyoda-ku, Tokyo, Japan.

PUBLISHED BY PACIFIC JOURNAL OF MATHEMATICS, A NON-PROFIT CORPORATION

The Supporting Institutions listed above contribute to the cost of publication of this Journal. but they are not owners or publishers and have no responsibility for its content or policies. 


\section{Pacific Journal of Mathematics}

\section{Vol. 14, No. $4 \quad$ August, 1964}

Homer Franklin Bechtell, Jr., Pseudo-Frattini subgroups . . . . . . . . . . . . . . . . 1129

Thomas Kelman Boehme and Andrew Michael Bruckner, Functions with convex means .............................................. 1137

Lutz Bungart, Boundary kernel functions for domains on complex manifolds . . . . . 1151

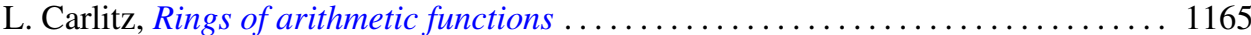

D. S. Carter, Uniqueness of a class of steady plane gravity flows . . . . . . . . . 1173

Richard Albert Dean and Robert Harvey Oehmke, Idempotent semigroups with

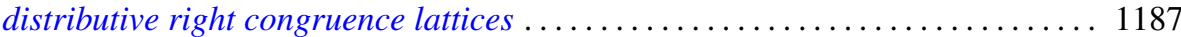

Lester Eli Dubins and David Amiel Freedman, Measurable sets of measures ..... . 1211

Robert Pertsch Gilbert, On class of elliptic partial differential equations in four

variables.......................................... 1223

Harry Gonshor, On abstract affine near-rings .................... 1237

Edward Everett Grace, Cut points in totally non-semi-locally-connected

continua ........................................... 1241

Edward Everett Grace, On local properties and $G_{\delta}$ sets ................ 1245

Keith A. Hardie, A proof of the Nakaoka-Toda formula . . . . . . . . . . . . . . . . 1249

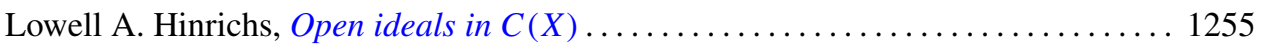

John Rolfe Isbell, Natural sums and abelianizing . . . . . . . . . . . . . . . . 1265

G. W. Kimble, A characterization of extremals for general multiple integral

problems............................................. 1283

Nand Kishore, A representation of the Bernoulli number $B_{n} \ldots \ldots \ldots \ldots \ldots \ldots \ldots \ldots$

Melven Robert Krom, A decision procedure for a class of formulas of first order predicate calculus.

Peter A. Lappan, Identity and uniqueness theorems for automorphic functions ...

Lorraine Doris Lavallee, Mosaics of metric continua and of quasi-Peano spaces . .

Mark Mahowald, On the normal bundle of a manifold..........

J. D. McKnight, Kleene quotient theorems

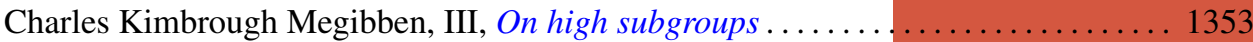

Philip Miles, Derivations on $B^{*}$ algebras . . . . . . . . . . . . . . . . . . . 1359

J. Marshall Osborn, A generalization of power-associativity . . . . . . . . . . . 1367

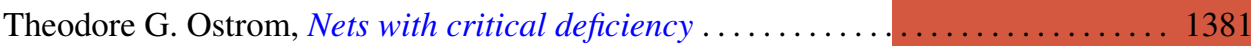

Elvira Rapaport Strasser, On the defining relations of a free product . . . . . . . . . 1389

K. Rogers, A note on orthoganal Latin squares . . . . . . . . . . . . . . . . . . 1395

P. P. Saworotnow, On continuity of multiplication in a complemented algebra ..... 1399

Johanan Schonheim, On coverings . . . . . . . . . . . . . . . . . . . . 1405

Victor Lenard Shapiro, Bounded generalized analytic functions on the torus . . . . . 1413

James D. Stafney, Arens multiplication and convolution . . . . . . . . . . . . . 1423

Daniel Sterling, Coverings of algebraic groups and Lie algebras of classical

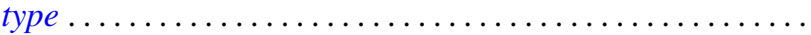

Alfred B. Willcox, Šlov type $C$ algebras over a connected locally compact abelian

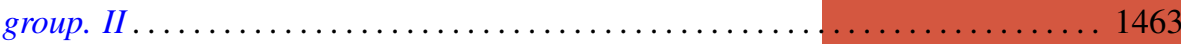

Bertram Yood, Faithful ${ }^{*}$-representations of normed algebras. II . . . . . . . . . 1475

Alexander Zabrodsky, Covering spaces of paracompact spaces 